\title{
Production en masse d'alevins mâles de Tilapia Oreochromis niloticus de la vallée du fleuve Sénégal à partir de l'aliment hormoné au 17 alpha méthyl testostérone
}

\author{
Momy SECK ${ }^{1}$, Hamet Diaw DIADHIOU ${ }^{2 *}$, Papa Demba NDAO ${ }^{3}$, \\ Théophile DIOUF ${ }^{4}$ et Abdoulaye NIANE ${ }^{5}$ \\ ${ }^{1}$ Antenne Nord de l'ANA à Richard Toll. \\ ${ }^{2}$ Institut Sénégalais de Recherches Agricoles / Centre de Recherches Océanographiques Dakar- \\ Thiaroye, BP2241 Dakar, Sénégal. \\ ${ }^{3}$ Centre de Recherche Biomédicale Espoir Pour La Santé (CRB/EPLS), Saint Louis, Sénégal. \\ ${ }^{4}$ Agence Nationale de l'Aquaculture / Antenne Nord Richard-Toll, Saint-Louis, Sénégal. \\ ${ }^{5}$ ANA, Dakar, Sénégal. \\ *Auteur correspondant; E-mail: hamet_diadhiou@yahoo.fr
}

\section{RESUME}

Cette étude a été réalisée en 2017 à la station de l'Agence Nationale de l'Aquaculture de Richard-Toll (vallée du fleuve Sénégal) dans le cadre du projet PISCI/FNRAA/WAAPP2 Commandité sur 1'Adaptation, Diffusion et Adoption de technologies de production en masse d'alevins mâles de Tilapia (Oreochromis niloticus) au Sénégal financé par le Fonds d'Impulsion de la Recherche Agro-Alimentaire du Sénégal (FNRAA). Face à un déficit d'alevins de tilapia confronté par les producteurs et à la difficulté de sexage manuel, ce travail œuvre à produire et fournir en quantité suffisante des alevins mono-sexe mâles de qualité aux producteurs et de transférer la technologie aux agences d'exécution de l'aquaculture et aux promoteurs privés. Pour ce faire, une activité de production en masse d'alevins mâles de la souche locale du Tilapia du Nil de la vallée du fleuve Sénégal a été testée en ajoutant l'hormone 17 alpha méthyl-testostérone dans l'aliment fourni à des alevins dont le sac vitellin vient d'être résorbé pendant 28 jours. Cette expérience a permis de produire 21168 alevins mâles de Tilapia (O. niloticus) en 3 mois et de cerner les limites de la production en masse d'alevins de la souche du Tilapia du Nil de la vallée du fleuve Sénégal au niveau de l'écloserie de l'ANA installée à Richard-Toll. Par conséquent, cette étude peut être reconduite en utilisant d'autres méthodes d'inversion sexuelle du Tilapia.

(C) 2018 International Formulae Group. All rights reserved.

Mots clés: Tilapia, Orechromis niloticus, 17 alpha méthyl-testostérone, inversion sexuelle, alevins mâles, aliment hormoné.

\section{Mass production of male fry of Tilapia Oreochromis niloticus from the Senegal River Valley from food supplemented with the 17 alpha methyl-testosterone}

\begin{abstract}
This study was carried out in 2017 at the station of the National Aquaculture Agency of Richard-Toll (Senegal River Valley) as part of the PISCI / FNRAA / WAAPP2 project on the Adaptation, Diffusion and Adoption of Technologies Mass production of male Tilapia (Oreochromis niloticus) fry in Senegal financed by
\end{abstract}


the Senegalese Agribusiness Research Impulse Fund (FNRAA). In the face of a deficit of Tilapia fry faced by producers and the difficulty of manual sexing, the project is working to produce and supply sufficient qualities of monosexal fry to producers and to transfer the technology to aquaculture executing agencies and private developers. To do this, an activity of mass production of male fry of the local Nile Tilapia strain of the Senegal River Valley was tested by adding the hormone 17 alpha methyl-testosterone in the feed supplied to fry including the yolk sac has been resorbed for 28 days. This experiment produced 21168 Tilapia (O. niloticus) male fry in 3 months and identified the limits of the mass production of fingerlings of the Tilapia strain of the Nile of the Senegal River Valley at the level of hatchery at ANA in Richard-Toll. Therefore, this study may be repeated using other methods of sexual inversion of Tilapia.

(C) 2018 International Formulae Group. All rights reserved.

Keywords: Tilapia, Oreochromis niloticus, 17 alpha methyl-testosterone, sexual inversion, male fry, hormonal food.

\section{INTRODUCTION}

La baisse des potentialités de pêche et la forte demande en poisson des populations ont amené l'Etat du Sénégal à s'intéresser au développement de la pisciculture à partir des années 2000, a inscrit l'aquaculture parmi les activités économiques à développer (MPEM, 2011).

Pour matérialiser cette volonté, il a été créé successivement des services pour asseoir la promotion de ce secteur (Direction de la Pêche Continentale et de l'Aquaculture (DPCA) devenue Direction de la Pêche Continentale (DPC), l'Agence pour la Promotion de l'Aquaculture (APA) devenue l'Agence Nationale de l'Aquaculture (ANA) et l'Agence Nationale de Retour vers l'Agriculture (AN REVA) devenue depuis 2009, l'Agence Nationale d'Insertion et de Développement Agricole (ANIDA). Dans les missions confiées à ces différentes structures, l'ANA a en charge la fourniture en quantité d'alevins de qualité, chose qu'elle ne parvenait pas à faire faute de savoir-faire et de moyens financiers. Le financement du Projet PAM Tilapia a permis à l'ANA d'avoir une écloserie moderne à Richard-Toll et de produire des alevins mâles de Tilapia en masse au profit des producteurs privés de la vallée du fleuve Sénégal. La production en masse d'alevins mâles de Tilapia en ajoutant l'hormone 17 Alpha méthyltestostérone est testée et adaptée à l'environnement de l'écloserie à poisson de l'ANA sise à RichardToll, dans le cadre de ce travail.

\section{MATERIEL ET METHODES Zone d'étude}

L'Agence Nationale de l'Aquaculture (ANA), Antenne Nord, est située dans la région de Saint-Louis, département de Dagana, commune de Richard-Toll (Figure 1). Elle est chargée de la mise en œuvre de la politique nationale de développement et de la promotion de l'aquaculture au Sénégal. Elle intervient dans le centre, la zone sud et la zone nord. Dans cette dernière, il existe 02 stations piscicoles et 01 écloserie : la station Piscicole de Thiabakh et la station de la Compagnie Sucrière Sénégalaise. C'est au niveau de l'écloserie que l'expérimentation s'est déroulée.

\section{Matériel}

Pour réaliser cette présente étude, nous avons utilisé : 6 bassins de reproduction de 10 $\mathrm{m}^{3}$ chacun suivant un ratio de 1 mâle pour 3 femelles; 10 bouteilles de Zoug pour l'incubation des œufs; 6 plateaux de résorption de la vésicule vitelline; 9 bacs en fibre de verre pour l'inversion des alevins; des appareils de mesure des paramètres physicochimiques à savoir un $\mathrm{pH}$-mètre pour mesurer le $\mathrm{pH}$ et un thermomètre pour la température; 1 logiciel (Google Earth) pour la localisation de la zone d'étude; Aliment de poisson en 
poudre de RAANAN FISH FEED avec un taux de protéine de $40 \%$ et un taux de conversion alimentaire de 1.3; de l'hormone 17alpha méthyle testostérone; de l'Ethanol 95\%; une balance électronique PHOCEE'NNE SF 400 capacité $7 \mathrm{~kg}$.

\section{Méthodes \\ Préparation des infrastructures d'élevage}

Les infrastructures d'élevage (bacs de reproduction) et le petit matériel de laboratoire sont nettoyés, désinfectés et mis à sec pendant au moins 48 heures avant d'y placer les géniteurs de tilapia. Les poissons sont triés par sexe à partir de l'observation de leurs papilles génitales. La papille génitale du mâle est allongée (gonopode) alors que celle de la femelle est courte et présente en son milieu une fente transversale (l'oviducte) située entre l'anus et l'orifice urétral (Figure 2). Ce qui permet de distinguer à l'œil nu, sans le microscope ou la loupe binoculaire, le mâle, de la femelle. Cette identification a permis de sélectionner pour l'étude 210 femelles et 72 mâles pour la reproduction et de les stockés dans des bassins en béton de 10 $\mathrm{m}^{3}$. Leur poids individuel moyen de ces géniteurs était de $236 \mathrm{~g}$ pour les femelles et de $250 \mathrm{~g}$ pour les mâles.

\section{Reproduction des géniteurs}

Six (06) bassins en béton de $10 \mathrm{~m}^{3}$ de volume chacun, répartis en 3 lots avec un ratio de 03 femelles pour 01 mâle ont servi pour la reproduction des poissons de poids moyen égal à $200 \mathrm{~g}$. Le premier lot est constitué par les bassins numéros 1 et 02 avec 40 femelles et 14 mâles dans chaque bassin. Le deuxième renferme les bassins 3 et 4 avec 35 femelles et 12 mâles dans chacun. Le troisième lot est constitué par les bassins 5 et 6 avec 30 femelles et 10 mâles dans chacun.

La reproduction des géniteurs est lancée dans ces différents lots avec un décalage de 2 jours par lot et elle a duré environ 10 jours du 16 mai au 20 juin 2017. Les géniteurs sont alimentés 3 fois par jour
( $09 \mathrm{~h}, 13 \mathrm{~h}$ et $17 \mathrm{~h}$ ) avec une ration de $3 \%$ de la biomasse.

\section{Suivi de la qualité de l'eau}

Le suivi de la qualité de l'eau s'effectuait avec la mesure des paramètres physico-chimiques à savoir la température mesurée à l'aide d'un thermomètre (JBL 0-40 ${ }^{\circ} \mathrm{C}$ ) suivant une fréquence de 3 fois par jour ( 9 $\mathrm{h}, 13 \mathrm{~h}$ et $17 \mathrm{~h}$ ). Le $\mathrm{pH}$ est mesuré une fois par jour à l'aide d'un pH-mètre Ecosens $10 \mathrm{~A}$. Les données sont enregistrées quotidiennement dans des fiches de suivi des paramètres physico-chimiques des géniteurs, ensuite reportées dans une base de données sur Excel.

\section{Collecte des oufs}

Suivant une température de reproduction de $29 \pm 2{ }^{\circ} \mathrm{C}$, la collecte des œufs s'est effectuée tous les 9 à 10 jours après. Ainsi, nous avons procédés à la pêche des géniteurs et à la collecte des œufs en incubation dans la bouche de la femelle. Les œufs collectés sont incubés artificiellement dans des bouteilles de Zoug.

\section{Préparation de l'aliment hormoné}

Une quantité de $240 \mathrm{ml}$ d'éthanol est mélangé à 60 mg de 17 Alpha méthyl testostérone. Un kilogramme d'aliment en poudre est pesé et ajouté à ce mélange jusqu'à obtenir quelque chose d'homogène. Ce produit est séché dans un endroit aéré à l'abri du soleil pendant 48 heures, puis conservé au réfrigérateur à $4{ }^{\circ} \mathrm{C}$ le temps d'être utilisé.

\section{Inversion hormonale des alevins}

Les alevins de poisson sont alimentés 6 fois par jour à $25 \%$ de leur biomasse totale durant les 10 premiers jours après la résorption de leur vésicule vitelline. Entre le 11 et le $20^{\text {ème }}$ jours, la quantité d'aliment fourni, est ramené à $20 \%$ de la biomasse. Du $21^{\text {ème }}$ au $28^{\text {ème }}$ jour, elle ne sera que de $15 \%$.

\section{Traitement de données collectées}

Les données enregistrées dans les fiches de suivi journalières sont saisies et traitées sur Excel et Word. 


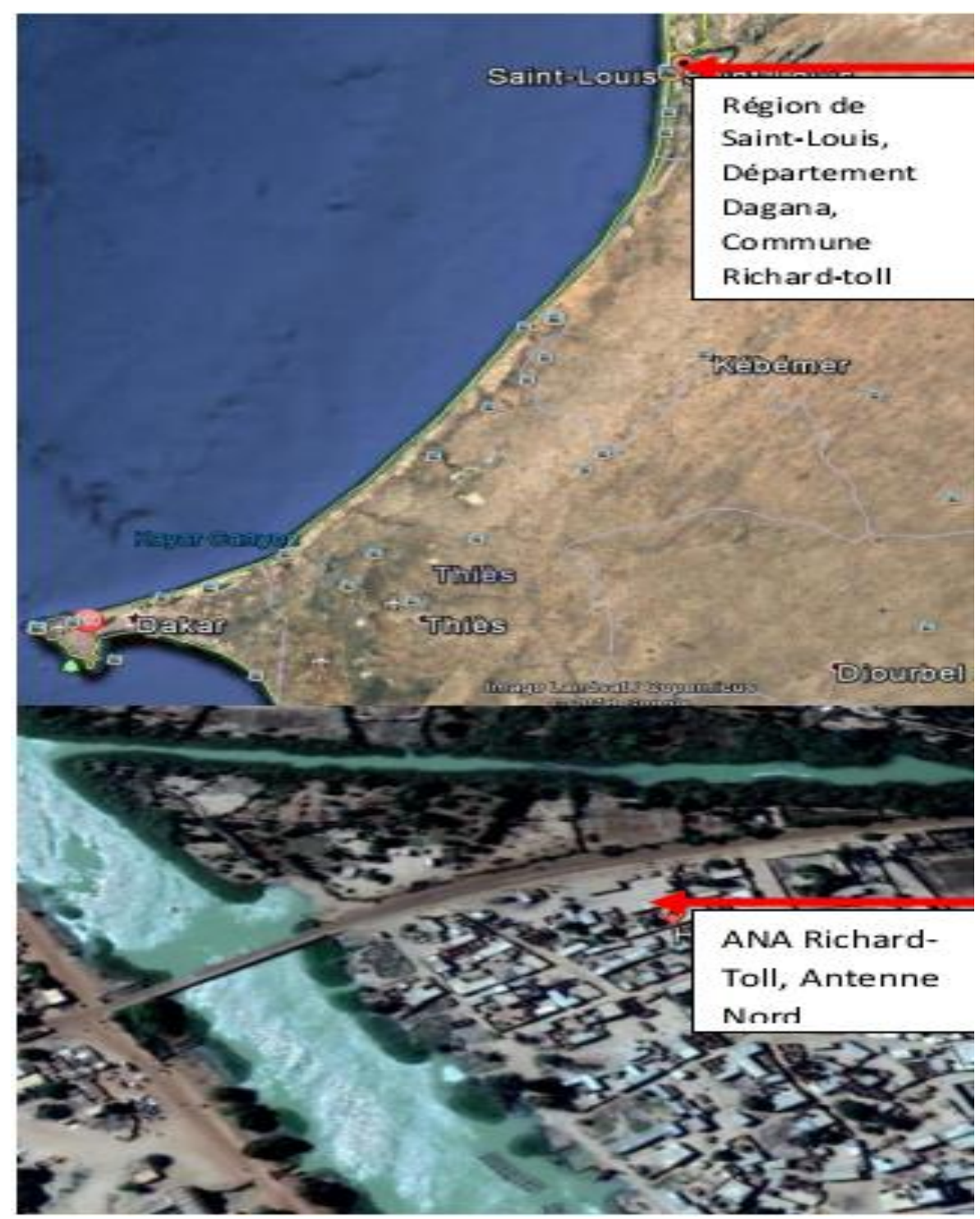

Figure 1 : Carte de localisation de la zone d'étude antenne Nord ANA Richard-Toll.
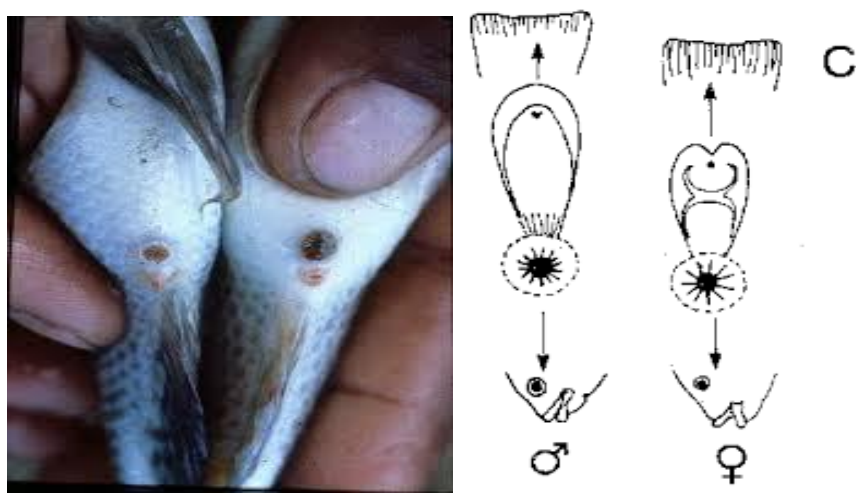

Figure 2 : Identification des géniteurs mâles et femelles (HAMOUDA, 2005). 


\section{RESULTATS}

\section{Reproduction et élevage des larves}

Suivant le stade des œufs, la durée d'incubation en moyenne est de 2 jours et celle de la résorption de la vésicule vitelline 3 jours suivant une température moyenne de 29 ${ }^{\circ} \mathrm{C}$. Après résorption de la vésicule vitelline, les larves sont récoltées et ensuite transférées vers les bacs d'inversion hormonale. Durant l'expérience, quarante-mille (40000) larves ont été produites.

\section{Alevinage}

Environ quarante-mille (40000) alevins ont été produits et nourris avec l'aliment hormoné pendant 28 jours suivant une fréquence de 6 fois par jour pour une ration alimentaire de $25 \%$ de la biomasse du $1^{\mathrm{er}}$ au $10^{\text {ème }}$ jour, ensuite $20 \% \mathrm{du} 11^{\text {ème }}$ au $20^{\text {ème }}$ jour et enfin $15 \%$ du $21^{\text {ème }}$ au $28^{\text {ème }}$ jour. Cependant, les alevins sont répartis dans 10 bacs en fibre de verre de $2 \mathrm{~m}^{3}$. Dans chacun de ces bacs, une densité de 2000 alevins au $\mathrm{m}^{3}$ est réparti d'où un nombre de 4000 alevins par bac. Après trois (3) mois d'alevinage, un nombre de 21168 alevins mâles inversés sur les quarante-mille (40000) initial est obtenu, ce qui nous donne un taux de survie d'environ $53 \%$. Des échantillonnages sont effectués pour déterminer le taux d'inversion par observation directe de la papille génitale et par squash gonadique sur un nombre de 100 individus par bassin. Après 28 jours d'inversion, les alevins sont estimés puis transférés dans sept (7) bassins en béton de 10 $\mathrm{m}^{3}$. Un nombre total de vingt et un mille cent soixante-huit (21168) alevins mâles ont été produits après trois (3) mois d'alevinage. Le taux de réussite de l'inversion est estimé égal à $97 \%$ de mâles pour les bassins 1,2 et 5 et $96 \%$ pour les bassins $3,4,6$ et 7 . Cependant, un faible taux de femelles après inversion estimé égal à $3 \%$ pour les bassins 1,2 et 5 , et $4 \%$ pour le reste. Ainsi, une douzaine d'alevins inversés ont atteint un poids moyen de $200 \mathrm{~g}$ en deux mois quinze-jours (Figure 3).

La Figure 4 représente le nombre d'alevins dans chaque bassin ainsi que leur poids moyen. Dans le bassin 1, six cent sept (607) alevins inversés sont estimés avec un poids moyen $40 \mathrm{~g}$, dans chacun des bassins 3 , 5, 6, un nombre de 2279 alevins de poids moyen $8 \mathrm{~g}$, dans celui du bassin 4, 2200 alevins de poids moyen $10 \mathrm{~g}$ et au niveau du bassin 7, un nombre de 9277 alevins de poids moyen $2 \mathrm{~g}$. Ainsi, une douzaine de tilapia inversés de poids moyen $200 \mathrm{~g}$ sont obtenus dans un happa de $4 \mathrm{~m}^{2}$ dans le bassin 1 .

Le Tableau 1 indique les différents intervalles des paramètres physico-chimiques durant la production d'alevins mâles inversés.

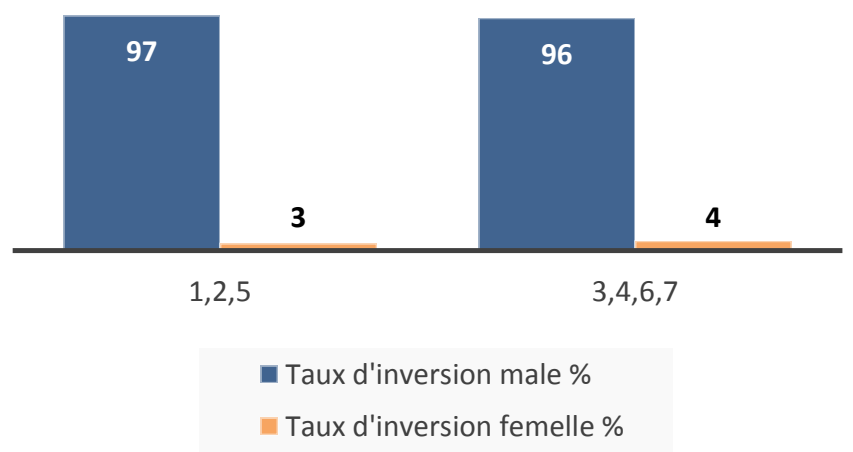

Figure 3: Taux d'inversion des alevins par bassin. 


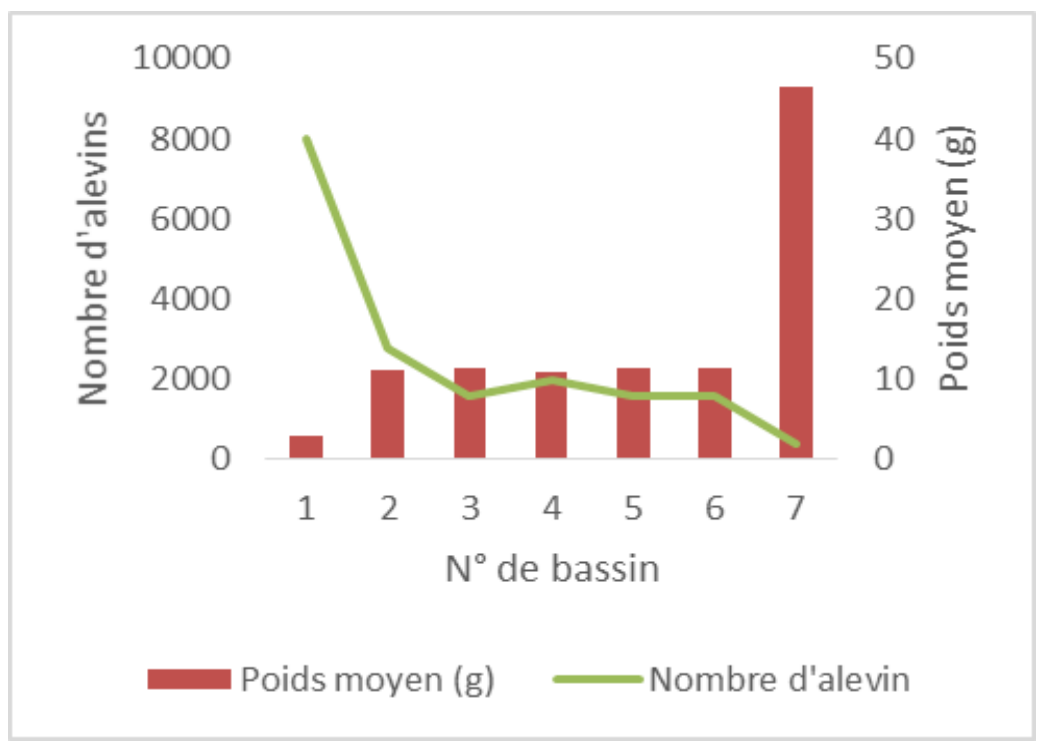

Figure 4: Nombre d'alevins et poids moyen par bassin.

Tableau 1: Paramètres physico-chimiques des différents bacs.

\begin{tabular}{lll}
\hline Bacs & $\mathbf{T}^{\circ} \mathbf{C}$ & $\mathbf{p H}$ \\
\hline Reproduction & $28-32$ & $7.9-8.5$ \\
Ecloserie & $27-29$ & $7-7.2$ \\
Inversion & $28-29$ & $7.3-7.8$ \\
Alevinage & $28-31$ & $7.5-8.2$ \\
\hline
\end{tabular}

\section{DISCUSSION}

\section{Complexités expérimentales}

Durant l'expérimentation, la difficulté majeure rencontrée est la fécondation asynchrone des géniteurs, ce qui favorise l'obtention d'alevins de poids moyen différent. Ouedraogo (2009) stipule « qu'une des difficultés rencontrées dans la mise en place des expérimentations réside dans le regroupement de cohortes issues de couples différents dont les fécondations ont eu lieu de façon asynchrone. Les effets parentaux peuvent induire des biais au niveau des résultats, notamment au niveau de la croissance ». Egalement, on peut noter la perturbation de la température de 2 à $4{ }^{\circ} \mathrm{C}$ due à l'inexistence d'un circuit fermé avec des thermorégulateurs ainsi qu'un système de filtration.

\section{Inversion hormonale}

Vingt et un mille cent soixante-huit alevins mâles inversés ont été produits sur 6 mois (mai à octobre 2017). Ceci montre l'efficacité de l'hormone 17 alpha méthyletestostérone sur les alevins. Cependant, le dosage de $60 \mathrm{mg}$ de 17 alpha méthyle testostérone par $\mathrm{kg}$ d'aliment appliqué pendant 28 jours révèle une efficacité variable des taux d'inversion en fonction des bassins (96 et $97 \%$ de mâles). Barry et al. (2007); Green et Teichert-Coddington (2000) ont rapporté que plus de 95\% des alevins de tilapias étaient masculinisés en 21-28 jours, lorsque les larves se nourrissaient avec 30-60 mg de $17 \alpha-\mathrm{MT} / \mathrm{kg}$ d'aliment. Dans notre 
étude, un taux important d'alevins inversés (96 et 97\%) a été obtenu avec la dose de 60 mg de MT / kg d'aliment. Senghor (2017) stipule que ses résultats ont indiqué que certaines doses de 17 $\alpha$-MT (60 mg de MT) semblent avoir un effet androgénique plus élevé sur les alevins de Oreochromis niloticus plutôt que d'autres doses. Ces résultats, concordent avec ceux de Barry et al. (2007) qui ont rapporté que plus de $95 \%$ des alevins de tilapias étaient masculinisés en 21-28 jours, lorsque les larves se nourrissaient avec 30-60 mg de $17 \alpha-\mathrm{MT} / \mathrm{kg}$ d'aliment. Ces résultats peuvent être dus aux difficultés de pouvoir maîtriser la température, le $\mathrm{pH}$ et également le renouvellement continu de l'eau sans système de filtration. Ce qui entraine une diminution de l'ingestion d'aliment hormoné. Il semble que la dose utilisé au cours de notre production est efficace pour induire une inversion de sexe.

\section{Taux de survie et croissance}

Au cours de l'expérience, nous avons enregistré des taux de survies faibles. Ceci pourrait être dû aux difficultés de maîtriser les paramètres physico-chimiques de production cités plus haut. Les mortalités sont plus importantes durant les 28 jours de l'inversion hormonale que durant l'alevinage. Piferrer (2001) précise que le taux de mortalité est plus fort dans les premiers stades de développement, lors des traitements hormonaux et aurait tendance à se stabiliser dans les stades avancés de l'ontogenèse. Senghor (2017) rapporte dans son étude, la potentialité de différentes doses $(30 ; 60$ et 90 mg de 17a-MT / kg d'aliment) qui a été étudiée et comparée au groupe témoin pendant les 28 premiers jours de l'alimentation. Elle démontre également l'effet androgénique de la MT dans le développement des caractères sexuels masculins qui a été confirmé avec 98\% de mâles dans le lot 1, traité avec $30 \mathrm{mg}$ de MT; $100 \%$ de mâles dans le lot $2(60 \mathrm{mg})$ et $96 \%$ pour le lot 3 traité avec $90 \mathrm{mg}$ de MT contre une moyenne assez équilibrée pour le témoin (52\% de mâles et $48 \%$ de femelles). Cependant, ces résultats ont indiqué que certaines doses de 17a-MT (60 mg de MT) semblent avoir un effet androgénique plus élevé sur les alevins de Oreochromis niloticus plutôt que d'autres doses.

De manière générale, la croissance des alevins inversés est assez forte, des poissons de $200 \mathrm{~g}$ en deux mois quinze-jours. Little et al. (2003) ont comparé les performances de l'élevage de différentes souches de Oreochromis niloticus et ont constaté que compte tenu de toutes les souches, le traitement à la MT des poissons a entrâné une taille finale de $10,7 \%$ de plus que les poissons non traités. Ceci est en concordance avec la croissance rapide des Tilapia de $200 \mathrm{~g}$ obtenus. Ces derniers, tous mâles, ont été mis dans un happa afin de minimiser les possibilités de cannibalisme.

\section{Conclusion}

L'objectif de cette étude est de fournir en quantité suffisante des alevins mono-sexe mâles de qualité aux producteurs et de transférer la technologie aux agences d'exécution de l'aquaculture et aux promoteurs privés. L'étude réalisée à Richard Toll a permis de produire 21168 alevins mâles inversés. Cette production aurait pu être dépasser si l'écloserie était équipée en station de pompage et filtration permanentes des eaux du fleuve, de thermostat pour réguler la température de l'eau dans les bacs de conditionnement des géniteurs, de groupes électrogènes pour assurer la fourniture continue en électricité, l'acquisition d'aliment de qualité et de meilleures souches de tilapia. Par conséquent, l'étude peut être reconduite en utilisant d'autres méthodes d'inversion sexuelle.

\section{CONFLIT D'INTERETS}

Les auteurs déclarent qu'il n'y a aucun conflit d'intérêts pour cet article.

\section{CONTRIBUTIONS DES AUTEURS}

MS, HDD et PDN ont aidé l'auteur principal à effectuer cette recherche; ADD a 
supervisé le travail et MS et PDN ont rédigé cet article.

\section{REMERCIEMENTS}

Les auteurs remercient le projet FNRAA d'avoir financé cette étude, les agences d'exécution des projets aquacoles, les institutions de recherches et les producteurs aquacoles locaux pour leurs contributions dans nos travaux de recherches.

\section{REFERENCES}

Barry TP, Marwah A, Marwah P. 2007. Stability of 17 a methyltestosterone in Fish Feed Aquaculture, 271: 523-529.

Green, Teichert-Coddington. 2000. Concentration of $17 \alpha$-Methyltestosterone in Hormone Treated Feed: Effects of Analytical Technique, Fabrication, and Storage Temperature. Journal of the World Aquaculture Society, 31(1). DOI: https://doi.org/10.1111/j.17497345.2000.tb00696.x

Hamouda IA. 2005. Contribution à l'étude de l'inversion sexuelle chez une espèce de poisson d'eau douce : Tilapia (Orechromis niloticus). Institut des sciences de la mer et de l'aménagement du littoral, France. p 5.

Lazard J. 2007. Transfert de poissons et développement de la production piscicole. Exemple de 3 pays d'Afrique Subsaharienne. Hydrobiologie Tropicale, 23 :251-265.

Little DC, Bhujel RC, Pham TA. 2003. Advanced nursing of mixed-sex and mono-sex tilapia (Oreochromis niloticus) fry, and its impact on subsequent growth in fertilized ponds. Aquaculture, 221 (2003): 265-276.

Ministère de la Pêche et de l'Economie Maritime, 2011. Document introductif du conseil interministériel sur la pêche, 54 p. MPEM, Sénégal.

Ouedraogo CRN. 2009. Inversion hormonale du sexe par le Méthyl testostérone et l'éthynyl oestradiol chez le Tilapia Oreochromis niloticus L. DEA Gestion Intégrée des Ressources Naturelles (GIRN), CIRDES, pp 34-35.

Piferrer F. 2001. Endocrine sex control strategies for the feminization of teleost fish. Aquaculture, 197 : 229-281.

Senghor ML. 2017. Impacts de l'utilisation de l'hormone $17 \alpha$ méthyl testostérone dans l'élevage du tilapia (Oreochromis niloticus, Linné 1758), sur le poisson de taille marchande et l'environnement. $\mathrm{PhD}$ Thèse. Ecole Inter-Etats des Sciences et Médecine Vétérinaire de Dakar, Université Cheikh Anta Diop de Dakar, p. 103. 